\title{
Study of Fabry-Perot Etalon Stability and Tuning for Spectroscopic Rayleigh Scattering
}

\author{
Michelle M. Clem* \\ NASA Glenn Research Center, Cleveland, Ohio, 44135 \\ Amy F. Mielke-Fagan ${ }^{\dagger}$ \\ NASA Glenn Research Center, Cleveland, Ohio, 44135 \\ Kristie A. Elam ${ }^{\ddagger}$ \\ Jacobs Sverdrup, Cleveland, Ohio, 44135
}

\begin{abstract}
[Abstract] The Fabry-Perot interferometer is a commonly employed instrument for resolving the spectrum of molecular Rayleigh scattered light for the purpose of evaluating flow properties such as gas velocity and temperature. Rayleigh scattered light from a focused laser beam can be directly imaged through a solid Fabry-Perot etalon onto a CCD detector to provide the spectral content of the scattered light. The spatial resolution of the measurements is governed by the locations of interference fringes. The location of the fringes can be changed by altering the etalon's physical characteristics, such as thickness and index of refraction. For a fused silica solid etalon the physical properties can be adjusted by changing the etalon temperature; hence changing the order of the interference pattern and the physical fringe locations. Controlling the temperature of the etalon can provide for a slow time-response spatial scanning method for this type of etalon system. A custom designed liquid crystal Fabry-Perot (LCFP) can provide for a fast time-response method of scanning the etalon system. Voltage applied to the liquid crystal interface sets the etalon's properties allowing Rayleigh measurements to be acquired at varying spatial locations across the image of the laser beam over a very short time period. A standard fused silica etalon and a tunable LCFP etalon are characterized to select the system that is best suited for Rayleigh scattering measurements in subsonic and supersonic flow regimes. A frequency-stabilized laser is used to investigate the apparent frequency stability and temperature sensitivity of the etalon systems. Frequency stability and temperature sensitivity data of the fused silica and LCFP etalon systems are presented in this paper, along with measurements of the LCFP etalon's tuning capabilities. Rayleigh scattering velocity measurements with both etalon systems are presented, in an effort to determine which etalon is better suited to provide optical flow measurements of velocity, temperature, and density.
\end{abstract}

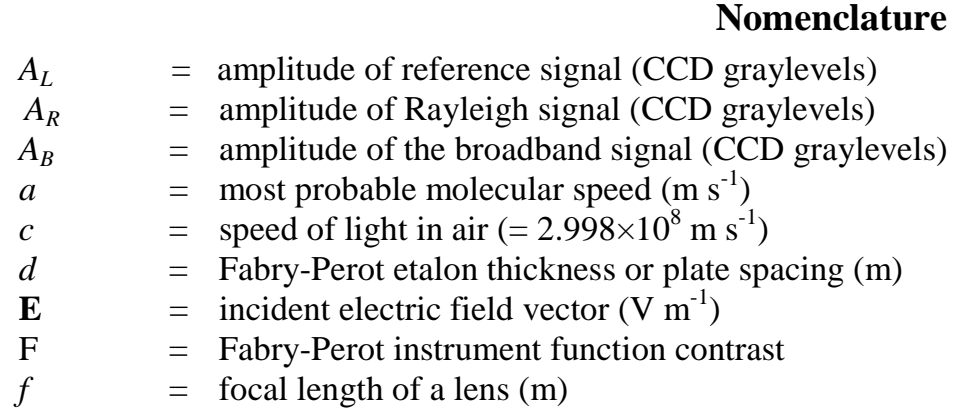

\footnotetext{
${ }^{*}$ Research Engineer, Optical Instrumentation and NDE Branch, 21000 Brookpark Rd/M.S. 77-1

${ }^{\dagger}$ Research Engineer, Optical Instrumentation and NDE Branch, 21000 Brookpark Rd/M.S. 77-1, AIAA Member

${ }^{\ddagger}$ Optics Technician, Optical Instrumentation and NDE Branch, 21000 Brookpark Rd/M.S. 77-3
} 


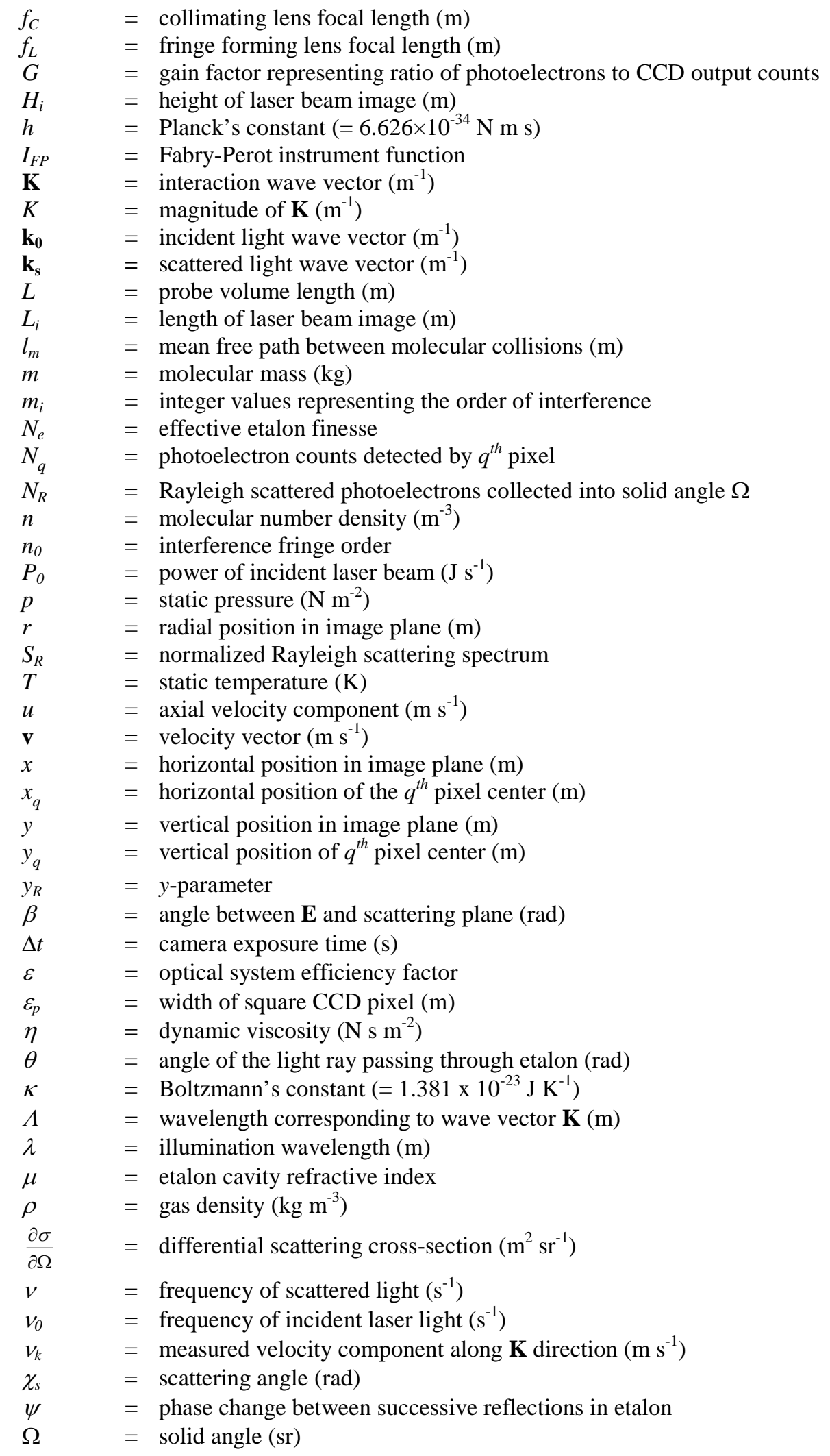




\section{Introduction}

$\mathbf{R}$ ayleigh scattering is an optical diagnostic technique that uses elastically scattered light from gas molecules to provide density, velocity, and temperature measurement ${ }^{1-8}$. One way to determine these properties from the scattered light is to analyze the signal strength, Doppler frequency shift, and spectral linewidth of the scattered light, which directly relate to density, velocity, and temperature, respectively. The Fabry-Perot interferometer (FPI) is one type of instrument that can be used to evaluate these spectral properties. A planar FPI consists of two parallel planar reflective surfaces. The cavity between the two surfaces can be either air or a solid transparent material, such as fused silica. When the spacing is fixed the instrument is often referred to as a Fabry-Perot etalon. When laser light is directed through the Fabry-Perot etalon the resulting interference pattern consists of an unequally-spaced concentric ring pattern that contains the spectrum of the light. The radial position of the interference fringes is a function of the frequency of the light and the etalon's plate thickness and index of refraction.

Past work has involved using an air-spaced FPI to resolve the Rayleigh scattering spectrum ${ }^{1-4}$. However, in harsh conditions and environments it is advantageous to use a solid etalon, since vibrations can change the plate spacing of an air-spaced interferometer and corrupt the spectral measurements. Some recent studies concerning Rayleigh scattering have utilized the solid etalon in place of the air-spaced interferometer ${ }^{5-7}$. It is often desirable to adjust the positions of the interference fringes or to maintain a fixed radial position of the fringes for various reasons. For spatially-resolved measurements, the measurement locations at the probe volume are dependent on the interference fringe positions in the detection plane. Therefore, tuning or scanning of the fringe positions is important to setting the measurement locations in the flow field. Also, maintaining stability of the fringe positions during data acquisition is critical to the velocity measurement accuracy ${ }^{9}$. The fringe positions can be changed by adjusting the etalon spacing or the index of refraction of the medium in the cavity. In the case of a solid etalon, both of these properties can be changed by adjusting the temperature of the etalon, however standard fused silica etalons lack the fast tunability that is available with air-spaced interferometers equipped with piezoelectric transducers that quickly change the mirror spacing. Liquid crystal Fabry-Perot (LCFP) etalons are now available which contain a thin liquid crystal layer in conjunction with glass layers to form the solid etalon cavity. Historically, LCFP's have been used as tunable optical filters in the infrared region for communications purposes ${ }^{11-12}$, however for the work presented in this paper, the LCFP is characterized and used in a Rayleigh scattering experiment. A custom designed LCFP was built by Scientific Solutions in which the liquid crystal index of refraction could be varied from 1.5 to 1.75 by adjusting the voltage applied to the liquid crystal allowing the etalon properties to be rapidly changed.

Both a fused silica solid etalon and a LCFP etalon are tested to determine which can provide the most stable interferometry and desired temperature sensitivity system for use in Rayleigh scattering experiments. In addition, the tuning capabilities of the LCFP etalon are presented as well as sample Rayleigh scattering data from a free jet flow, acquired with both the fused silica and LCFP etalon systems.

\section{Rayleigh Scattering and Fabry-Perot Interferometry}

In molecular Rayleigh scattering an incident electric field interacts with an atom or a molecule inducing a dipole moment that oscillates and radiates at the frequency of the incident field. It is considered an elastic scattering process because the internal energy of the molecule is unchanged and the frequency of the light is changed only by the Doppler effect due to the thermal as well as the bulk motion of the molecules ${ }^{8}$. The frequency spectrum of the scattered light contains information about the gas density, bulk velocity, and temperature. Fig. 1 shows a Rayleigh scattering spectrum containing the narrow laser line and a typical Rayleigh spectral peak to illustrate how the flow property measurements are obtained from the spectral information. If the gas composition is fixed, the total intensity of the Rayleigh scattered light is directly proportional to the gas density. The frequency shift between the laser peak and the Rayleigh peak is proportional to the bulk flow velocity. The width of the spectrum is related to the gas temperature.

The shape of the spectrum is dependent on gas pressure $p$ and temperature $T$, and the scattering angle $\chi_{s}^{13}$. A nondimensional parameter $y_{R}$, which represents the ratio of the wavelength of the scattering grating $\left(\Lambda=\frac{\lambda}{2 \sin \frac{\chi_{s}}{2}}\right)$ to the mean free path between molecular collisions $\left(l_{m}=\frac{a \eta}{{ }_{n} T}\right)$, is used to establish spectral shape regimes: 


$$
y_{R}=\frac{\Lambda}{2 \pi l_{m}}=\frac{p}{\eta K a}
$$

where

$$
a=\sqrt{\frac{2 \kappa T}{m}}
$$

The measured velocity component, $v_{k}$, is in the same direction as the interaction wave vector $\mathbf{K}$, which is the bisector of the incident and scattered light wave vectors, $\mathbf{k}_{\mathbf{0}}$ and $\mathbf{k}_{\mathbf{s}}$, respectively (Fig. 2). The interaction wave vector and its magnitude $K$ are given by:

$$
\begin{gathered}
\mathbf{K}=\mathbf{k}_{\mathbf{s}}-\mathbf{k}_{\mathbf{0}} \\
K=|\mathbf{K}|=\frac{4 \pi}{\lambda}\left[\sin \frac{\chi_{s}}{2}\right]
\end{gathered}
$$

The geometry of the optical arrangement in an experiment is designed such that the desired component of the velocity vector $\mathbf{v}$ is measured:

$$
v_{k}=\frac{\mathbf{K} \cdot \mathbf{v}}{K}
$$

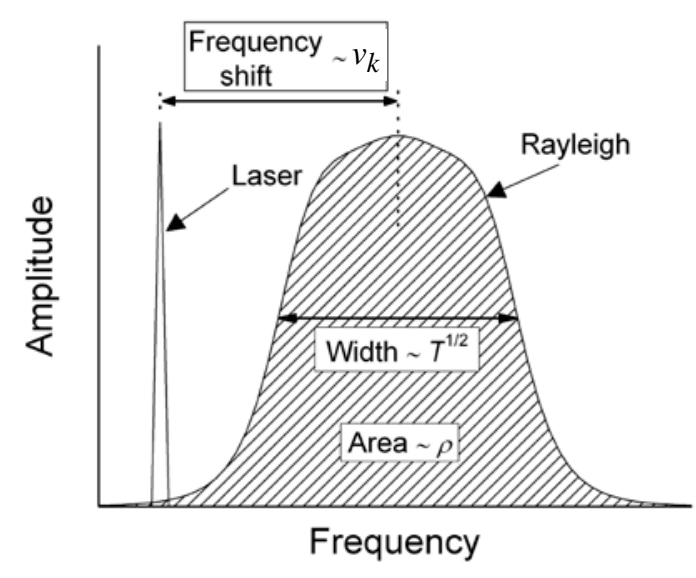

Fig. 1 Rayleigh scattering spectrum.

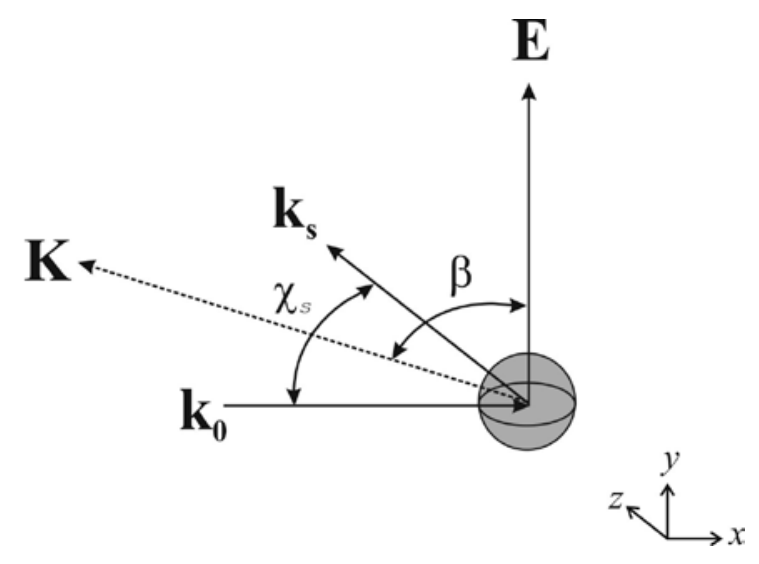

Fig. 2 Light scattering from a moving particle.

A planar Fabry-Perot etalon can be used to analyze the spectrum of Rayleigh scattered light ${ }^{9}$. A solid FP etalon consists of a solid optical material with parallel planar reflective surfaces having a fixed thickness. The interference created between the reflective plates creates standing waves, which generate constructive interference when the wavelength matches the optical path length. Therefore, maximum transmission occurs when the optical path-length difference $(O P D)$ between each transmitted beam is equal to an integer multiple of the wavelength, or

$$
O P D=2 \mu d \cos \theta=m_{i} \lambda
$$


where $m_{i}$ are integer values representing the order of interference. The maximum reflectivity, or minimum transmission occurs when the $O P D$ is equal to half of an odd multiple of the wavelength. By varying either the etalon thickness $(d)$, the index of refraction $(\mu)$ of the optical medium in the cavity between the reflective surfaces, or the angle of the incoming light ray $(\theta)$, transmission of different frequencies will occur and recreate the Rayleigh spectrum. When the etalon thickness and the medium refractive index are held constant, while the incoming light ray $\theta$ is varied by imaging points off-axis, the etalon is said to be operated in the static "imaging mode". A lens at the etalon output images the light source at the image plane where a detector is located, as shown in Fig. 3 . If the incoming light has a very narrow line width, the imaged intensity pattern is essentially a delta function convolved with the Fabry-Perot instrument function defined as:

$$
I_{F P}=\frac{1}{1+F \sin ^{2} \frac{\psi}{2}}
$$

where $\mathrm{F}$ is the contrast:

$$
F=\frac{1}{\sin ^{2} \frac{\pi}{2 N_{e}}}
$$

$N_{e}$ is the effective etalon finesse and $\psi$ is the phase change of light between successive reflections:

$$
\psi=\frac{4 \pi \mu d}{\lambda} \cos \theta
$$

Fig. 4 shows a typical fringe interference pattern resulting from a planar single-frequency light source being passed through the solid etalon. For spectrally broadened light, such as Rayleigh scattered light, the spectrum of the light is convolved with the Fabry-Perot instrument function and the fringes broaden accordingly; the line width of the fringes provides a measure of temperature from Rayleigh scattered light. In this work, the light source is molecular scattered light from a focused laser beam, which provides a linear interference pattern, such as that shown in Fig. 5. The frequency shift of the light associated with the bulk flow velocity is determined by a spatial shift in the fringe positions in the image.

The fringes generated by the FP etalon have unequal spacing that is directly related to the free spectral range (FSR) of the instrument, which is the change in frequency needed to shift the system by one fringe and can be evaluated by the following relation:

$$
\mathrm{FSR}=\frac{c}{2 d \mu}
$$

where $c$ is the speed of light. Adjusting the etalon thickness or the index of refraction changes the FSR, thus shifting the fringe spacing and locations. In the case of the standard solid etalon, a change in the material temperature results in a change of etalon FSR due to the fact that $d$ and $\mu$ are both a function of temperature. In this work the fringe positions are monitored as the room temperature is changed to evaluate the stability of the system in a harsh environment with unstable temperature. Fig. 6 shows a linear profile of a FP interference pattern like the one shown in Fig. 4 for several values of etalon thickness, $d$. The thickness only has to change by a fraction of a wavelength to produce a noticeable shift in the fringe locations; therefore it only takes a very small temperature change on the order of $0.01^{\circ} \mathrm{C}$ to produce a noticeable shift in fringe location. In the case of the LCFP, the supplied voltage is 
adjusted to change the etalon properties. Voltage adjustments can be used to tune the fringes to the desired measurement locations. Both the solid etalon and the LCFP etalon are studied and characterized in this work to assess which is best suited for acquiring Rayleigh scattering measurements in wind tunnels and other test facilities at NASA Glenn Research Center.

In the experiments using both etalon systems Rayleigh scattered light is collected from a finite length $L$ of the laser beam and imaged at the CCD detector. The expected number of Rayleigh photoelectron counts incident on the detector plane without the FP etalon in the optical path can be expressed as:

$$
\left\langle N_{R}\right\rangle=\int_{\Delta \Omega} \frac{\varepsilon P_{0} n L \lambda \Delta t \frac{\partial \sigma}{\partial \Omega} \sin ^{2} \beta}{h c} \partial \Omega
$$

where $\varepsilon$ is the overall system efficiency including detector quantum efficiency and other losses. With the interferometer in the optical path, the amount of energy, in terms of CCD gray level counts, collected on the $q^{\text {th }}$ pixel of the detector centered at position $\left(x_{q}, y_{q}\right)$ including light scattered from gas molecules (Rayleigh scattering), and broadband background light is expressed as:

$$
\left\langle N_{q, \text { Rayleigh }}\right\rangle=\frac{A_{R}}{\varepsilon_{p}^{2}} \int_{y_{q}-\frac{\varepsilon_{p}}{2}}^{y_{q}+\frac{\varepsilon_{p}}{2}} \int_{x_{q}-\frac{\varepsilon_{p}}{2}}^{x_{q}+\frac{\varepsilon_{p}}{2}} \int_{-\infty}^{+\infty} S_{R}\left(v, u_{k}\right) I_{F P}(v, x, y) d v d x d y+A_{B}
$$

where $A_{B}$ is the amplitude of the broadband background light and the amplitude of the Rayleigh scattered light is defined as:

$$
A_{R}=\left(\left\langle N_{R}\right\rangle \varepsilon_{p}^{2}\right) /\left(G L_{i} H_{i}\right)
$$

The Rayleigh spectrum $S_{R}$ is evaluated using the TENTI S6 kinetic theory model ${ }^{13,14}$. A reference image containing the interference pattern associated with light at the incident laser frequency is recorded within seconds of the Rayleigh image to provide the reference fringe radius, which is needed to obtain an accurate velocity estimate from the Doppler frequency shift between the incident and molecularly scattered light. The model function for the energy collected on the $q^{\text {th }}$ pixel of the detector including scattered light at the laser frequency and background light is expressed by the following equation:

$$
\left\langle N_{q, \text { Reference }}\right\rangle=\frac{A_{L}}{\varepsilon_{p}^{2}} \int_{y_{q}-\frac{\varepsilon_{p}}{2}}^{y_{q}+\frac{\varepsilon_{p}}{2}} \int_{x_{q}-\frac{\varepsilon_{p}}{2}}^{x_{q}} I_{F P}\left(v_{0}, x, y\right) d x d y+A_{B}
$$

The imaged fringe patterns are analyzed by maximum likelihood estimation (MLE) analysis ${ }^{15}$ using the model functions described in Eq. (12) and Eq. (14) to extract the velocity and temperature information at multiple spatial points in the flow field simultaneously. 


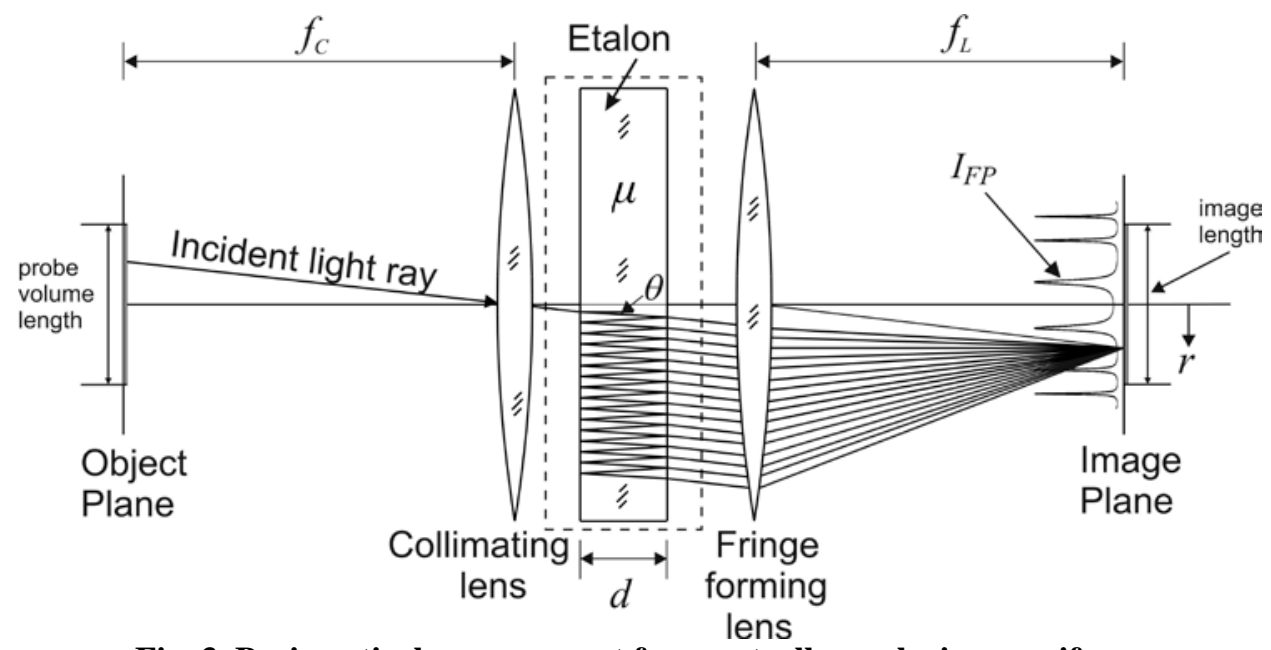

Fig. 3 Basic optical arrangement for spectrally-analyzing a uniform light source using a solid Fabry-Perot etalon.

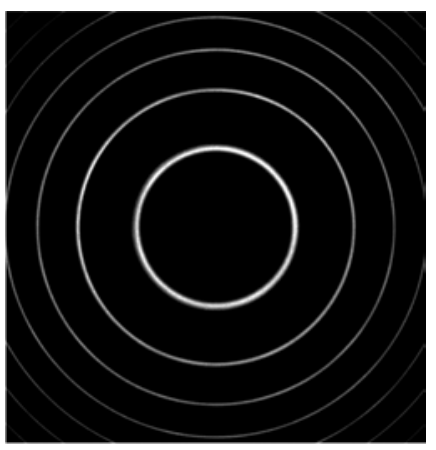

Fig. 4 Concentric ring interference pattern from a planar singlefrequency light source imaged through a Fabry-Perot etalon.

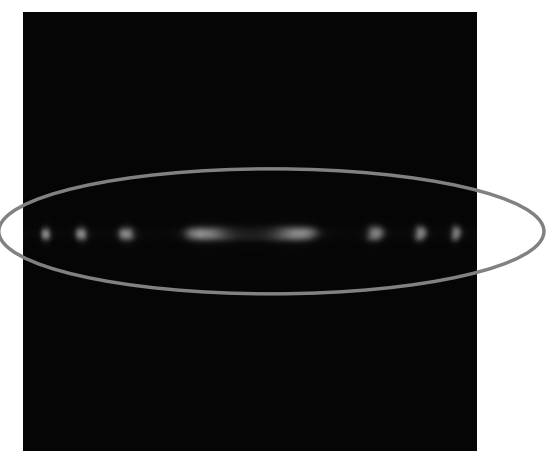

Fig. 5 Rayleigh light scattering from a focused single-frequency laser beam imaged through a Fabry-Perot etalon.

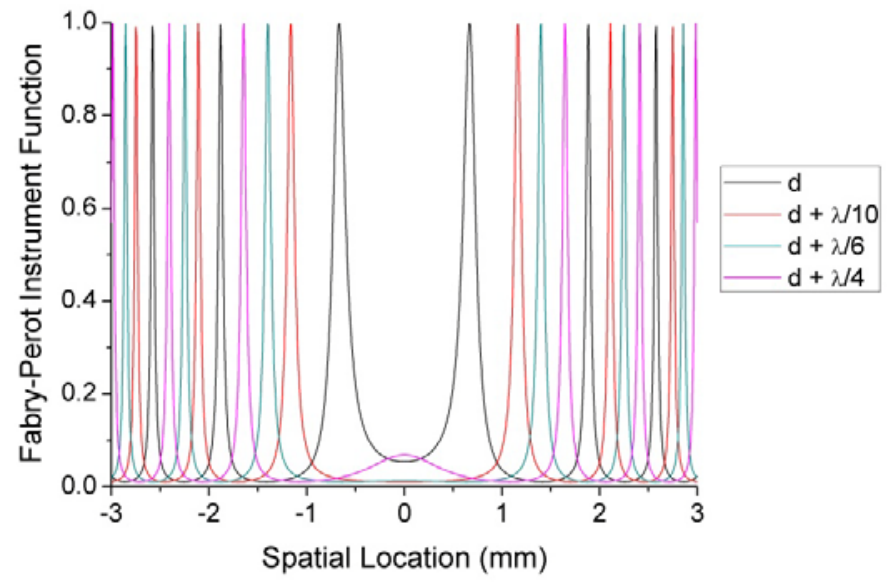

Fig. 6 One-dimensional Fabry-Perot instrument function profiles for several etalon thickness values, where $d$ $=10.22 \mathrm{~mm}$ and $\lambda=532 \mathrm{~nm}$. 


\section{Experimental Setup}

\section{A. Etalon stability and temperature sensitivity testing}

Testing of both the fused silica etalon and the LCFP in terms of effective frequency stability and temperature change sensitivity under various room temperature conditions was performed. A $200 \mathrm{~mW}$ frequency-stabilized 532 $\mathrm{nm}$ laser source was used to provide the light source for the optical system. This light source had frequency stability of approximately $\pm 3 \mathrm{MHz}$. A diagram of this system is shown in Fig. 7. A $20 \mathrm{~mm}$ lens (Lens 1) was placed directly in front the laser output to defocus the light onto a diffuser screen. The surface of the diffuser acted as the object plane of the optical system. A $150 \mathrm{~mm}$ achromatic focal length lens (Lens 2) collimated the scattered light from the diffuser surface and directed it through the specified etalon. The $25 \mathrm{~mm}$ diameter fused silica etalon had a refractive index of approximately 1.46 and $90 \%$ reflectivity coating that provided a reflective finesse of 30 . The etalon was approximately $11.3 \mathrm{~mm}$ thick giving a FSR of $9 \mathrm{GHz}$. The $25 \mathrm{~mm}$ diameter LCFP etalon also had $90 \%$ reflectivity coatings giving a reflective finesse of 30 . The index of refraction of the LCFP system was variable from 1.5 to 1.75 and the total etalon thickness was about $40 \mathrm{~mm}$ giving a FSR that ranged from 2.1 to $2.5 \mathrm{GHz}$. A 400 $\mathrm{mm}$ focal length Nikon lens (Lens 3) focused the image of the diffuser plane at the camera detector plane. The image of the illuminated diffuser had been modified by the transmission properties of the etalon resulting in a circular interference fringe pattern. A type $\mathrm{K}$ thermocouple was used to monitor the temperature in the fused silica etalon chamber. Due to the housing of the LCFP etalon, the thermocouple was not able to be placed in the etalon chamber, therefore it was placed on the outside of the housing measuring the room temperature. A program for data acquisition and analysis was developed using LabVIEW. Images were acquired from the camera and least-squares analysis was used to fit the instrument function described in Eq. (14) to the interference pattern. The motion of the fringes over time was used to provide a measure of apparent frequency stability and temperature change sensitivity of the system; for a perfectly stable system the fringes would remain at a fixed location at all times. Data was acquired for both etalon systems: a fused silica etalon and a LCFP etalon, both using the $200 \mathrm{~mW}$ frequencystabilized $532 \mathrm{~nm}$ laser as the light source.

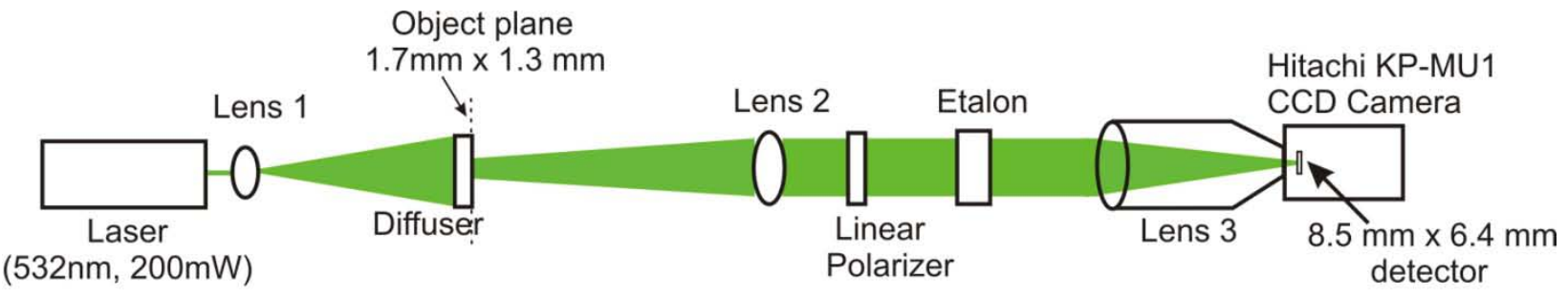

Lens 1: $20 \mathrm{~mm}$ focal length

Lens 2: $150 \mathrm{~mm}$ focal length

Lens 3: $400 \mathrm{~mm}$ focal length Nikon camera lens

Fig. 7 Diagram of optical system to study the effective frequency stability and temperature change sensitivity of two etalon systems.

The instability of the etalon in terms of apparent frequency change was monitored by tracking the radius $(r)$ of a fringe of the interference pattern with initial radius $r_{0}$ and order $n_{0}$. The apparent frequency change $(\Delta v)$ is related to the radius change of the fringe by the following analysis:

$$
\Delta v=\left[\left(\frac{r}{r_{0}}\right)^{2}-1\right] n_{0} F S R
$$




$$
n_{0}=\frac{d}{\mu \lambda}\left[\frac{r_{0}}{f_{L}}\right]^{2}
$$

where $f_{L}$ is the focal length of lens that focuses the fringe pattern at the image plane (i.e., Lens 3 in Fig. 7). The apparent frequency change was monitored for 90 minute periods, at three different temperature conditions (stable, increasing, and decreasing room temperature), for the two etalon systems.

\section{B. Rayleigh scattering measurements in a free jet}

Rayleigh scattering data from a free jet flow was separately acquired using the fused silica and LCFP etalon systems. A schematic of this set up can be seen in Fig. 8. A Coherent Verdi $18 \mathrm{~W}$ continuous-wave $532 \mathrm{~nm}$ laser with a $2.25 \mathrm{~mm}$ diameter output beam provided the incident light for the system. The vertically polarized laser beam was focused with a $200 \mathrm{~mm}$ focal length lens (Lens 1) to a diameter of $60 \mu \mathrm{m}$ at the probe volume. The beam was oriented at a $45^{\circ}$ angle to the primary flow direction, while the scattered light was collected at a $90^{\circ}$ scattering angle. The incident and scattering wave vectors were arranged such that the axial component of the jet velocity $u$ was measured, as indicated by the red $\mathbf{K}$ vector in Fig. 8. A $12 \mathrm{~mm}$ length of the laser beam was imaged through the etalon by a pair of $300 \mathrm{~mm}$ focal length lenses. An $f / 6$ achromat lens (Lens 2) collimated the light and directed it through the etalon system. A f/4 $300 \mathrm{~mm}$ focal length Nikon camera lens (Lens 3) then focused the image of the laser beam onto the detector of a Princeton Instruments VersArray back-illuminated, scientific-grade CCD camera. The image of the laser beam had been modified by the transmission properties of the etalon, resulting in Rayleigh scattered light at the location of the interference fringes. Stray light provided by placing a white sheet behind the probe volume was imaged through the etalon within seconds of acquiring the Rayleigh image. The imaged stray light provided reference laser image data for use in the frequency shift analysis of the acquired Rayleigh scattering images. Due to the ordinary and extraordinary states of the birefringent liquid crystal there are two sets of interference patterns that are transmitted simultaneously by the LCFP. One set is static while the other is tunable with voltage. The transmission of these two states is polarization sensitive. Therefore, a linear polarizer was placed in front of the LCFP etalon to block the non-tunable state from passing through the system. During both experiments, images of the Rayleigh light and reference light were acquired with 500 ms exposure time.

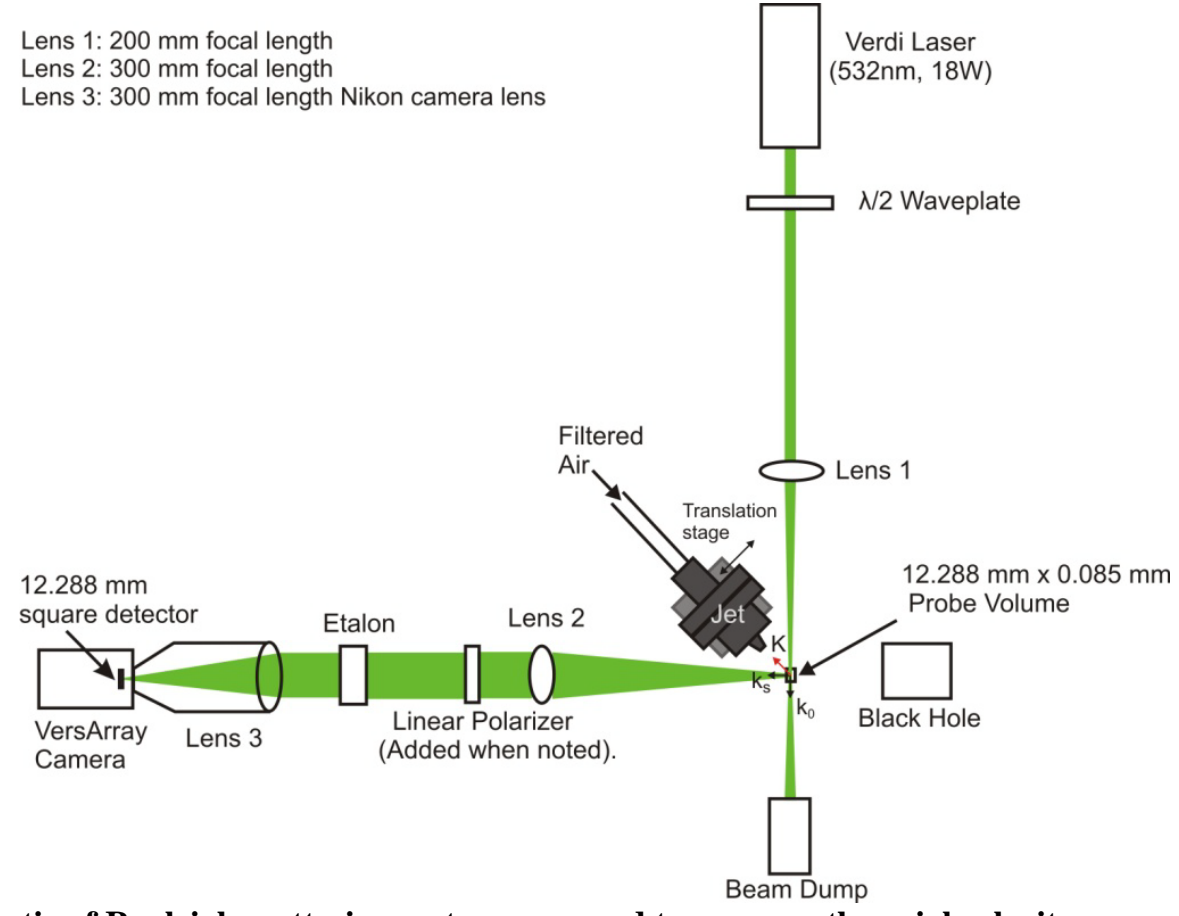

Fig. 8 Schematic of Rayleigh scattering system arranged to measure the axial velocity component of a free jet flow. 


\section{Results and Discussion}

\section{A. Etalon stability and temperature sensitivity}

Testing of etalon stability and temperature change sensitivity was performed using both the fused silica etalon and the LCFP etalon systems with the $200 \mathrm{~mW}$ frequency-stabilized $532 \mathrm{~nm}$ laser as the light source. Changing the etalon characteristics, such as the etalon thickness or the index of refraction, alters the location of the interference fringes. Due to the fact that the etalon thickness and the index of refraction are both related to the temperature of the etalon medium, changing the room temperature was changed to modified these characteristics. In order to tune or stabilize the etalon by temperature control, the sensitivity of the etalon to temperature change must be characterized. The location or radius of the innermost fringe of the interference pattern was used to provide a measure of the apparent frequency change of the etalon systems. Data was acquired separately for each etalon system over three different periods of room temperature control.

The first test was performed at a stable room temperature for a period of 90 minutes where the etalon temperature was stable to within approximately $\pm 0.1^{\circ} \mathrm{C}$. This was the baseline test to see how stable the etalons were in a moderately stable temperature environment. Figs. 9(a) and 9(b) show the etalon or room temperature (right vertical axis) and apparent frequency change in $\mathrm{MHz}$ (left vertical axis) over a 90-minute time period for moderately stable room temperature conditions for both the fused silica and the LCFP etalons. The apparent frequency change is measured relative to an arbitrary reference frequency. Although the temperature was considered to be "stable" the thermocouple measurements indicate that the temperature actually drifted up or down over these 90-minute assessment periods. This temperature change, although small, produced a noticeable effect in the apparent frequency of the etalon. It is important to note that due to physical constraints it was difficult to place the thermocouple within the LCFP housing as it was for the fused silica etalon. Therefore room temperature data, representative of the LCFP etalon temperature, is presented for the LCFP data. During the 90-minute stability assessment, the data shows the fused silica etalon demonstrated a total apparent frequency shift of approximately $300 \mathrm{MHz}$ while the etalon temperature drifted by about $0.1^{\circ} \mathrm{C}$. Alternatively, the LCFP etalon demonstrated apparent frequency drift of about $70 \mathrm{MHz}$ while the room temperature drifted downward by about $0.1^{\circ} \mathrm{C}$.
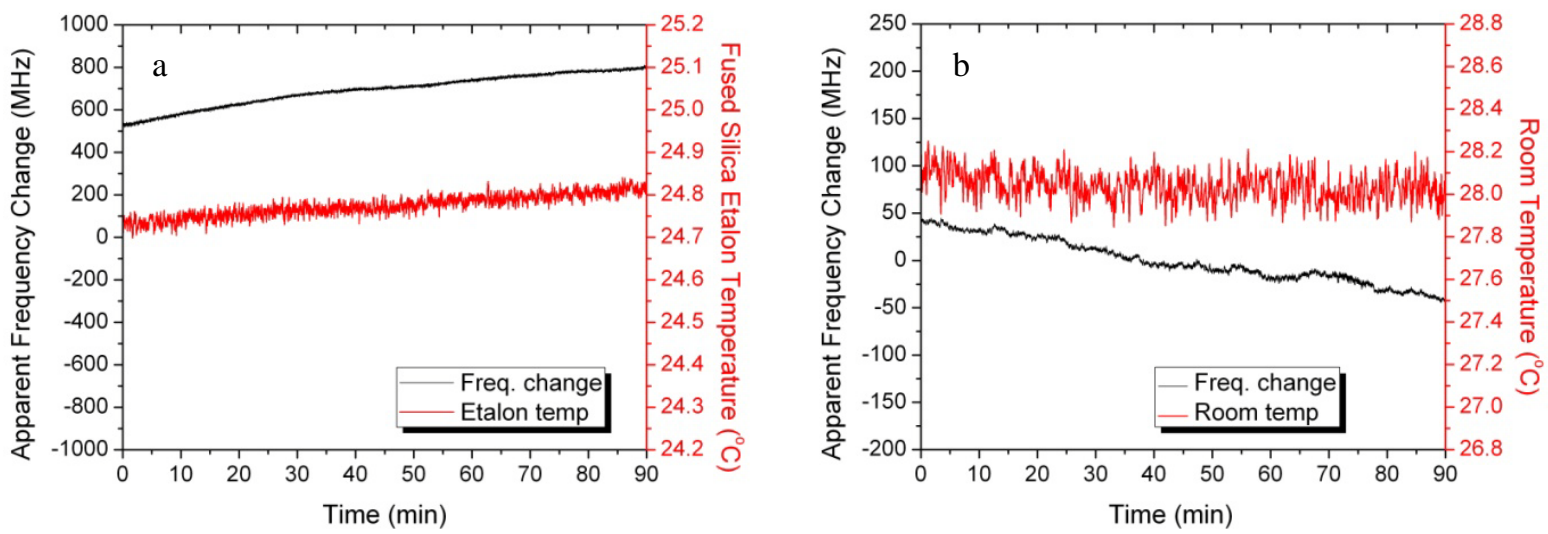

Fig. 9 Apparent frequency stability of the fused silica (a) and LCFP (b) etalon systems based on a frequency-stabilized laser source at a moderately stable etalon temperature.

The method often taken for Rayleigh data acquisition involves acquiring an image of Rayleigh scattered light and then acquiring a reference laser image approximately 15-30 seconds later. Therefore, it is important to know the short-term stability of the etalon system being used in the experiment because any instability of the etalon system (i.e. apparent frequency change) will result in an error in the velocity measurement. In order to assess the short-term stability of each etalon system a 1 minute period of the data collected in this first experiment is shown in Fig. 10. The short-term apparent frequency stability of the LCFP is $\pm 2 \mathrm{MHz}$ and the short-term apparent frequency stability of the fused silica etalon is $\pm 4 \mathrm{MHz}$. In a Rayleigh scattering experiment the velocity is calculated from the measured frequency change between the incident laser light and the Rayleigh scattered light, which is a function of the fringe shift of the laser and Rayleigh signal peaks, as illustrated in Fig. 11. A change in the etalon characteristics between the acquisition of each signal can bias the relative shift between the two signal peaks. The radii of the laser 
and Rayleigh fringe are used to calculate the relative frequency change via Eqs. (15) and (16). The relationship between the frequency change and velocity $v_{k}$ is:

$$
v_{k}=\frac{2 \pi \Delta f}{|K|}
$$

The LCFP etalon short-term instability of $\pm 2 \mathrm{MHz}$ could result in velocity bias error of as much as $1.5 \mathrm{~m} / \mathrm{s}$ and the fused silica etalon with instability of $\pm 4 \mathrm{MHz}$ could result in errors of as much as $3.0 \mathrm{~m} / \mathrm{s}$. However, Rayleigh scattering is typically used to measure high velocity flows where these errors would not be very significant.

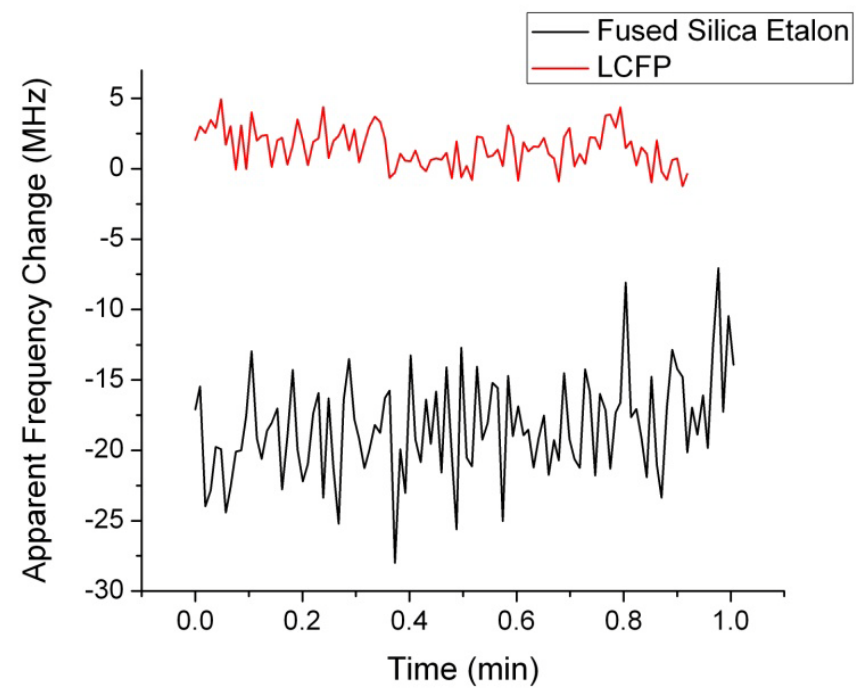

Fig. 10 Short-term apparent frequency stability of the fused silica etalon and LCFP etalon systems based on frequency-stabilized laser source at a stable room temperature.

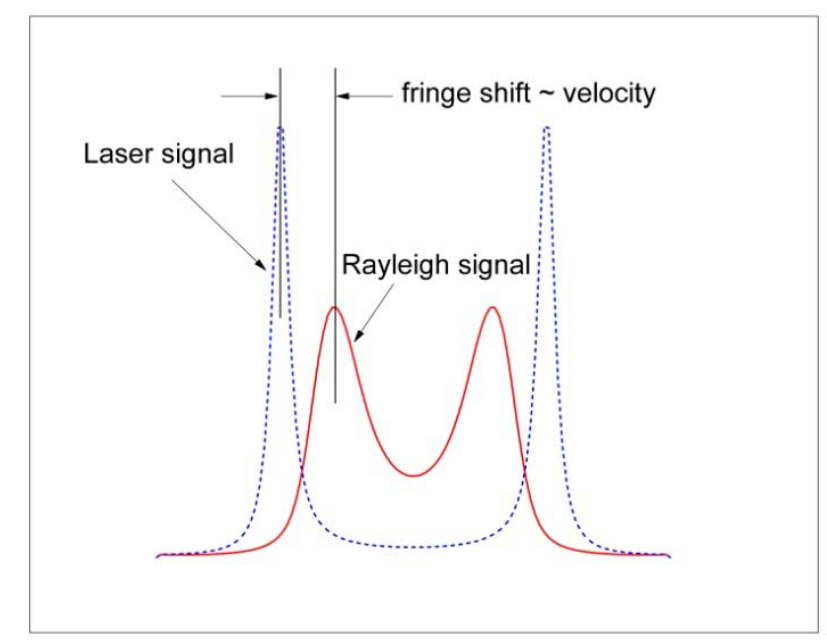

Fig. 11 Linear profile through the first-order fringe in a circular interference pattern obtained from imaging Rayleigh scattered light and single frequency reference light through the etalon system.

In the second test the room temperature was increased and data was acquired for approximately 90 minutes. The temperature and resulting apparent frequency change over the time period are shown in Figs.12(a) and 12(b) for the fused silica and LCFP etalons, respectively. The temperature of the etalon systems increased by approximately $0.5^{\circ} \mathrm{C}$ over the 90 -minute period. The data shows that the apparent frequency change of the fused silica etalon follows a very similar trend as the temperature change of the etalon. The apparent frequency change of the LCFP 
etalon does not follow as closely with the LCFP etalon temperature trend due to the fact that the temperature presented is the room temperature. The LCFP approaches frequency stability after 65 minutes, whereas the temperature stabilizes after 20 minutes. In both etalon systems the apparent frequency increases with increasing etalon temperature. The apparent frequency change was much greater for the fused silica etalon over approximately the same temperature change for both systems. The fused silica etalon demonstrated a total apparent frequency change of approximately $1685 \mathrm{MHz}$ over an increase of temperature of nearly $0.46{ }^{\circ} \mathrm{C}$, characterizing the etalon to have a temperature sensitivity of $3663 \mathrm{MHz} /{ }^{\circ} \mathrm{C}$. The LCFP had a total apparent change in frequency of $265 \mathrm{MHz}$ over an increase of $0.5^{\circ} \mathrm{C}$, giving the etalon a temperature sensitivity of $530 \mathrm{MHz} /{ }^{\circ} \mathrm{C}$.

In the third test the room temperature was decreased and data was collected separately for each etalon system for 90 minutes. The temperature and resulting apparent frequency change over the time period are shown in Figs. 13(a) and 13(b) for the fused silica and LCFP etalons, respectively. The apparent frequency change of both etalon systems decreased as the etalon temperature decreased. The fused silica etalon demonstrated an overall apparent frequency change of $1408 \mathrm{MHz}$ over a temperature change of $0.4^{\circ} \mathrm{C}$; thus temperature sensitivity of the etalon was $3520 \mathrm{MHz} /{ }^{\circ} \mathrm{C}$. The LCFP etalon demonstrated a total apparent frequency change of $350.3 \mathrm{MHz}$ over a temperature change of $0.6{ }^{\circ} \mathrm{C}$; giving a temperature sensitivity of $583 \mathrm{MHz} /{ }^{\circ} \mathrm{C}$ for the LCFP etalon. Overall, whether the temperature is increasing or decreasing, the fused silica etalon has an average temperature sensitivity of approximately $3600 \mathrm{MHz} /{ }^{\circ} \mathrm{C}$ while the LCFP etalon has an average temperature sensitivity of approximately 550 $\mathrm{MHz} /{ }^{\circ} \mathrm{C}$.
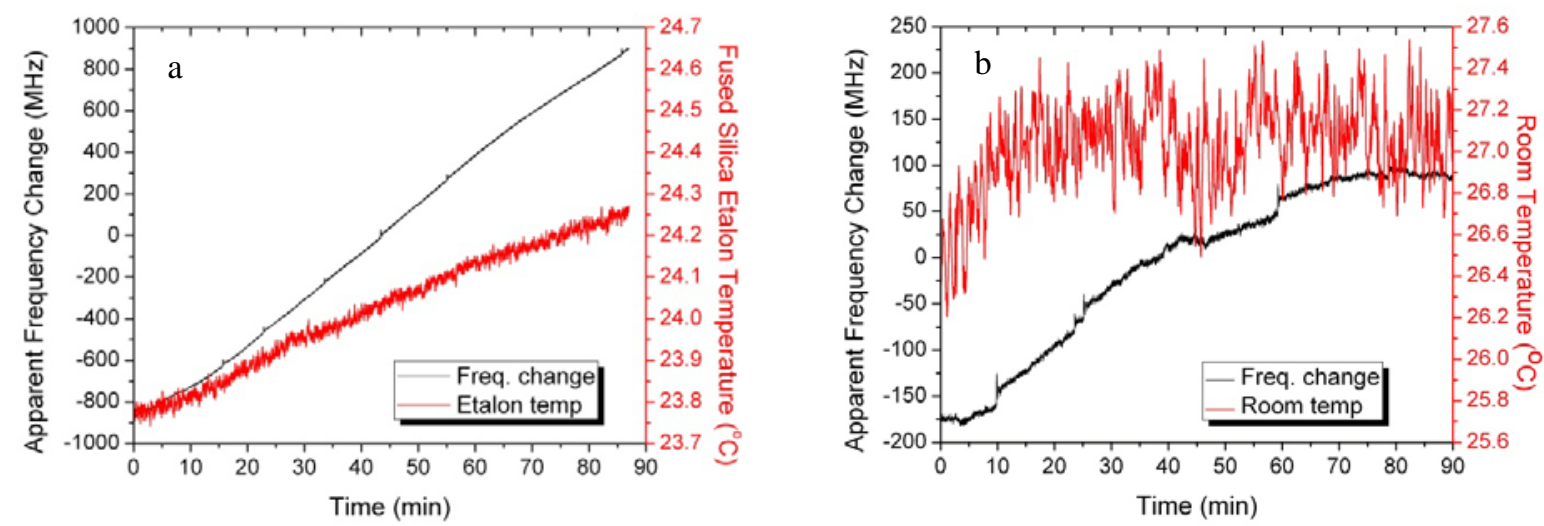

Fig. 12 Effective frequency stability of the fused silica etalon (a) and LCFP etalon (b) systems based on frequency-stabilized laser source at an increasing etalon temperature.

The apparent frequency stability of the fused silica etalon was measured to be $\pm 4 \mathrm{MHz}$ and the temperature sensitivity was measured to be $3600 \mathrm{MHz} /{ }^{\circ} \mathrm{C}$. This means that in order to make the fused silica etalon stable to within $\pm 4 \mathrm{MHz}$ or better in a harsh environment with large temperature fluctuations the etalon temperature needs to be controlled to $\pm 0.001{ }^{\circ} \mathrm{C}$. Typical temperature controllers are accurate within $0.1{ }^{\circ} \mathrm{C}$, making tuning of the etalon's physical characteristics (thickness and index of refraction) via temperature control very impractical. The same is true for the LCFP; its measured apparent frequency stability was $\pm 2 \mathrm{MHz}$ and its temperature sensitivity was $550 \mathrm{MHz} /{ }^{\circ} \mathrm{C}$. To hold the apparent frequency stable within $\pm 2 \mathrm{MHz}$ it is necessary to control the temperature within $\pm 0.003^{\circ} \mathrm{C}$, which again is not practical. 

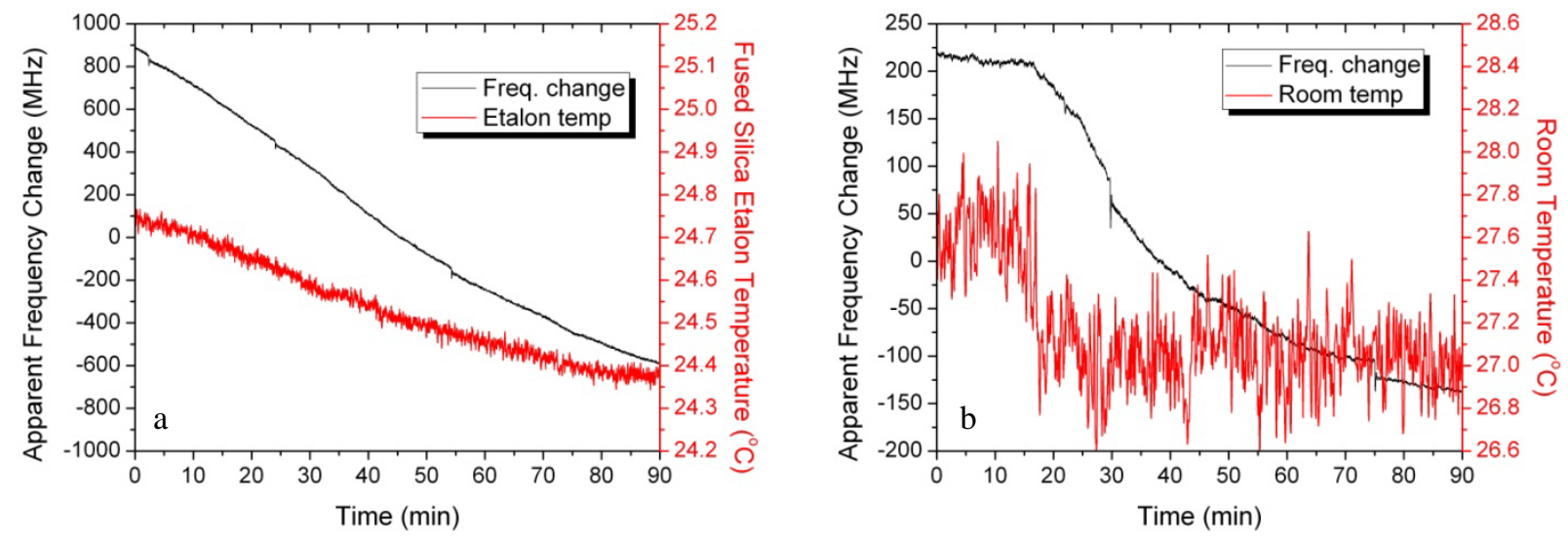

Fig. 13 Effective frequency stability of the fused silica etalon (a) and LCFP etalon (b) systems based on frequency-stabilized laser source at a decreasing etalon temperature.

\section{B. Liquid Crystal Tunability}

Due to its liquid crystal composition, the LCFP etalon has the capability of rapid tuning in order to adjust the location of the interference fringes, providing a fast time-response spatial scanning method for the etalon system. A voltage applied to the liquid crystal interface sets the etalon characteristics. The liquid crystal response time to a voltage change is on the order of milliseconds. In theory, the voltage can be scanned rapidly to provide Rayleigh measurements at varying spatial locations across the image of the laser beam over a short period of time, although this was not implemented in the Rayleigh measurements presented in this paper. In an effort to characterize the tuning capabilities, the voltage of the LCFP etalon was increased while the radius of one of the fringes was tracked using the same experiment setup as the etalon stability testing. Figure 14 shows the radius of the tracked fringe in terms of camera pixels (left vertical axis) and the corresponding apparent frequency change (right vertical axis) as a function of the voltage applied to the liquid crystal. As the voltage level of the LCFP was increased from 5.1 V to $9.7 \mathrm{~V}$, the radius of the tracked fringe decreased continuously as it cycled through more than one fringe order. The points labeled (a) through (f) in Fig. 14 refer to the images in Fig. 15 that correspond to those data points. The fringe that is being tracked is indicated by a yellow arrow in each image. At $9.7 \mathrm{~V}$ the radius of that fringe became too small to accurately track and it became necessary to begin tracking the next order fringe for data points (g) through (j) (corresponding fringe images not shown). Figure 14 shows that as the LCFP voltage is further increased from $10.1 \mathrm{~V}$ to $12.4 \mathrm{~V}$, the radius of the tracked fringe does not decrease as rapidly as it did while the LCFP voltage was increased from $5.1 \mathrm{~V}$ to $9.7 \mathrm{~V}$ due to the nonlinearity of the response of the liquid crystal. The slope for data points (a) through (f) is approximately 29 pixels/V while the slope for data points (g) through (j) is about 15 pixels/V. The tuning capability of the LCFP etalon will be utilized in future Rayleigh scattering work at NASA GRC. There are various reasons that one would want to tune the interference fringes. It is often necessary in Rayleigh experiments to set the diameter of the innermost fringe to a specific location such that it falls within the illuminated image region at the detector or to set the fringe diameter to a value that minimizes the measurement uncertainty ${ }^{9}$. The tuning feature may also be used in multiple-point Rayleigh measurement systems to change the physical locations of the measurements in the flow field, which are set by the interference fringe locations in the image ${ }^{7}$. This feature could also be used to stabilize the etalon system in a non-thermally-controlled testing environment where controlling the etalon temperature cannot provide the required stability, as mentioned earlier. 


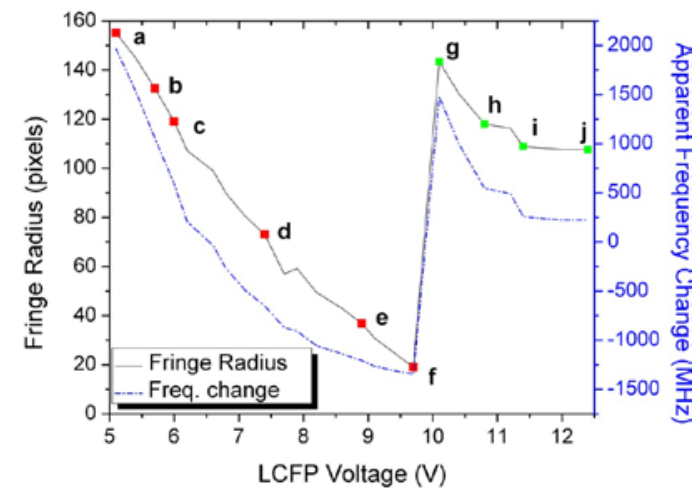

Fig. 14 Fringe radius and apparent frequency change as a function of LCFP voltage.

a

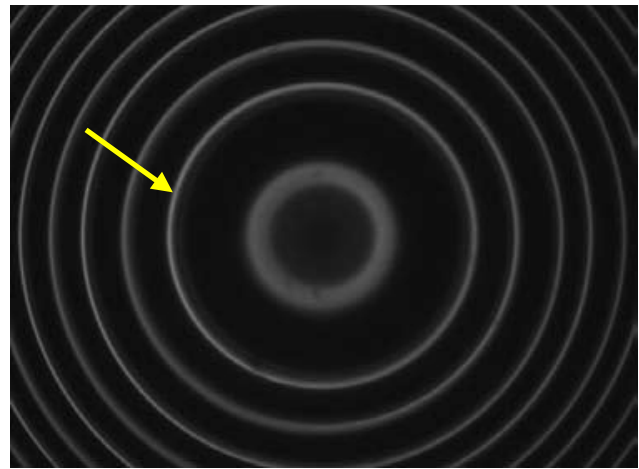

C

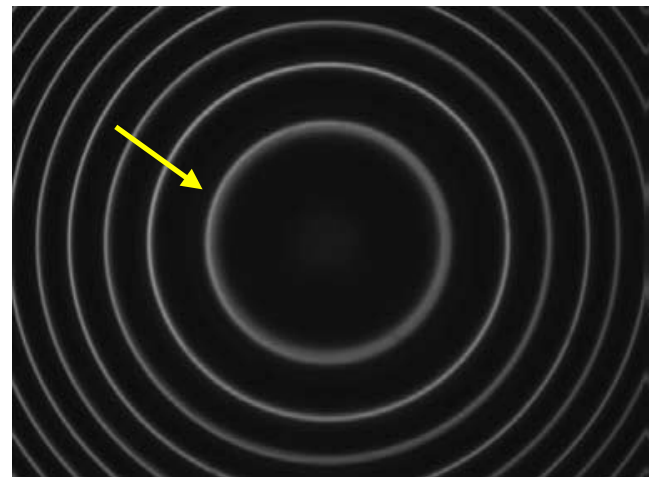

e

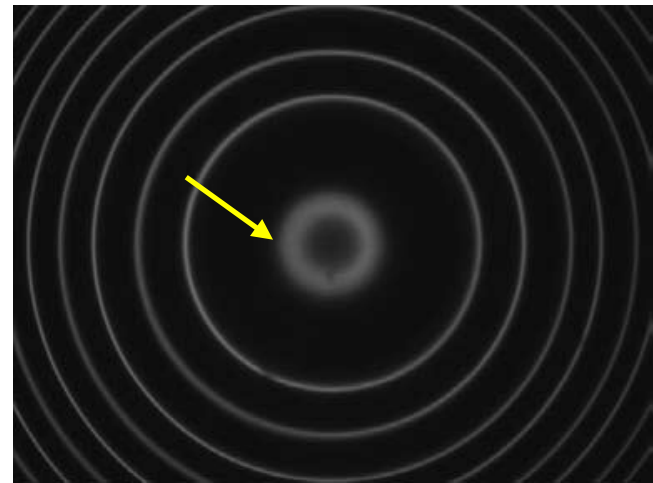

b

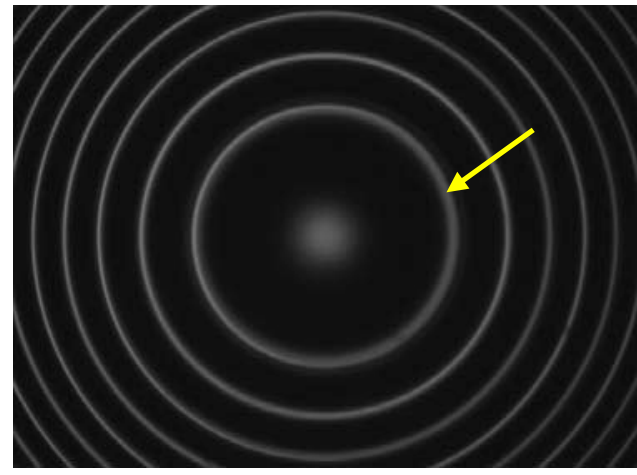

d

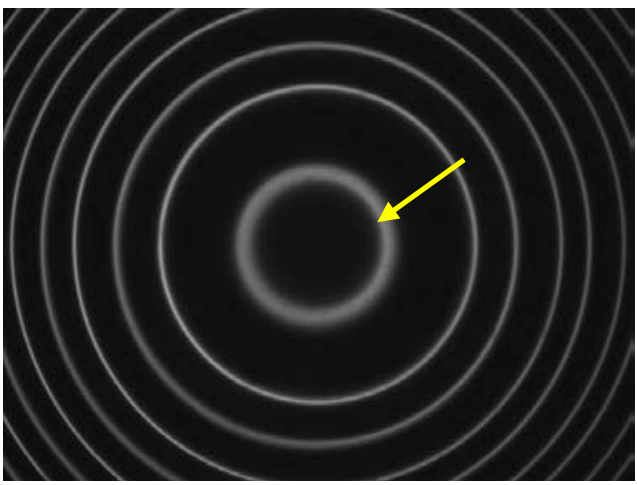

f

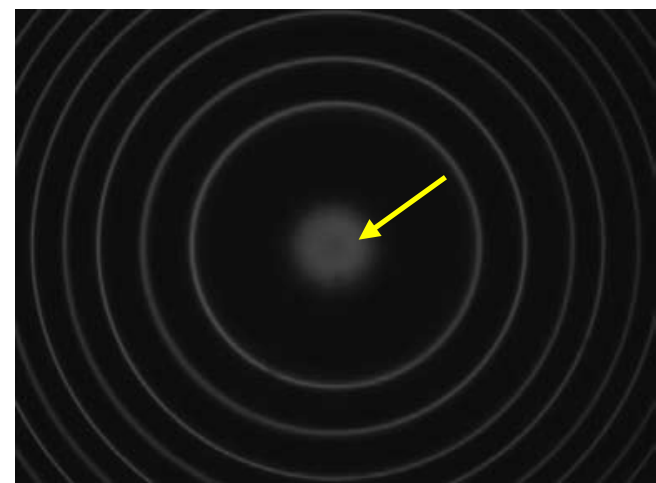

Fig. 15 Interference fringe images corresponding to data points (a) through (f) in Fig. 14 where the fringe indicated by the arrow was tracked as the LCFP voltage was changed from (a) $5.1 \mathrm{~V}$ to (f) $9.7 \mathrm{~V}$. 


\section{Rayleigh scattering measurements in a free jet}

Rayleigh scattering data from a free jet flow was acquired using the two different etalon systems. The jet exit velocity for the test involving the fused silica etalon was $193 \mathrm{~m} / \mathrm{s}$ and the exit velocity for the test using the LCFP etalon system was $187 \mathrm{~m} / \mathrm{s}$. In both tests, one image was acquired with pure Rayleigh signal followed closely by acquisition of an image where reference laser signal was scattered into the collection system by placing a white sheet behind the probe volume. The image data from the fused silica test is presented in Fig. 16 where Fig. 16(a) contains the pure Rayleigh signal and Fig. 16(b) contains both Rayleigh and reference laser signal. The image data from the LCFP etalon test is shown in Fig. 17 where the pure Rayleigh signal and the combined Rayleigh and reference signals are presented in Figs. 17(a) and 17(b), respectively. The Rayleigh signal is only present across a few horizontal rows of pixels since the signal comes from imaging the focused laser beam through the etalon; the fringes are only imaged in the locations where there is imaged signal. If a planar region of the flow was imaged the Rayleigh fringes would be circular just like the reference laser fringes in Figs. 16(b) and 17(b). The difference in FSR of the two systems is clearly evident by the large difference in the number of interference fringes between the images in Figs. 16(b) and 17(b) even though the systems were used in identical optical arrangements. The lower FSR of the LCFP etalon $(2.5 \mathrm{GHz})$ caused the fringes to be spaced more closely together than in the case of the fused silica etalon, which had a FSR of $9 \mathrm{GHz}$. The low FSR of the LCFP does not present a problem when resolving the narrow linewidth laser light; however the much broader Rayleigh spectrum, which is on the order of 1$2 \mathrm{GHz}$ wide, fills nearly the entire gap between fringes, making it difficult to differentiate between the individual Rayleigh fringes in Fig. 17(a). The overlap of each Rayleigh fringe with the adjacent orders made the analysis of the data very difficult and resulted in high measurement error. When analyzing the reference image from the LCFP test it was observed that some dust particles had been entrained in the flow during the process of holding a white sheet near the probe volume resulting in some particle scattering signal. Particle or Mie scattering results in a Doppler shifted signal associated with the motion of the particles in the flow just like Rayleigh scattering; however due to the larger size of the particles compared to molecules, the scattered light spectrum is not thermally broadened. The narrow Mie scattering peaks in the reference image were used to measure flow velocities in the LCFP data instead of using the Rayleigh signal in Fig. 17(a).
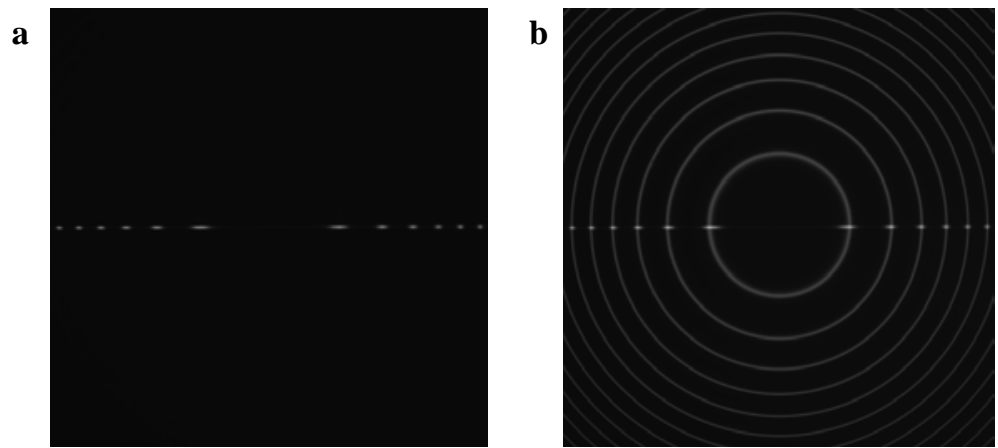

Fig. 16 Sample images of Rayleigh scattered light (a) and Rayleigh scattered light combined with reference light (b) for the fused silica etalon system.

a

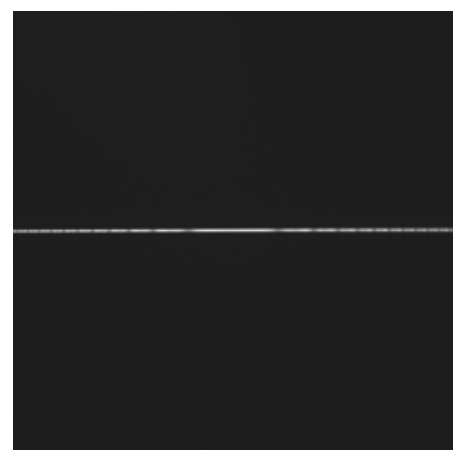

b

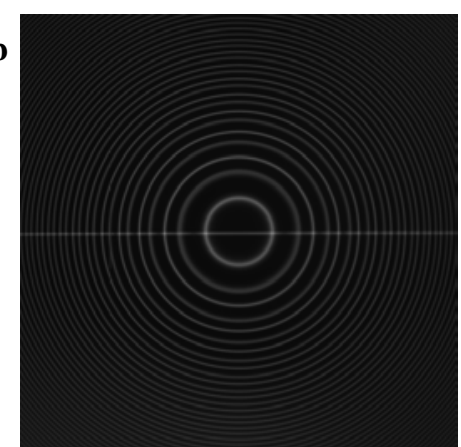

Fig. 17 Sample images of Rayleigh scattered light (a) and Rayleigh scattered light combined with reference light (b) for the LCFP etalon system. 
Figure 18 shows a linear profile across a 300 pixel region of the fused silica image data. The CCD values at those pixels are shown as black circles. The model function that was fit to the reference laser signal data by MLE analysis is shown by the blue curve and the model function that was fit to the Rayleigh signal is shown by the red curve. The radial shift between the reference and Rayleigh fringes is used to provide a measure of flow velocity at each fringe location. Almost the entire jet profile was mapped with a single snapshot in this experiment. Figure 19 shows similar data for the LCFP reference image data only. The combined reference laser and Mie signals are shown by the black circles and dashed line while the model function that was fit to the combined laser and Mie data is shown by the red curve. Only half of the LCFP fringes were analyzed since every other fringe order appeared to be wider than the adjacent fringes, which is evident in Fig. 17(b) and the line profile shown in Fig. 19. The increased fringe width made it difficult to differentiate between the laser and Mie signal peaks. The reason for the increased fringe width will be investigated further in the future.

The resulting velocity estimates from the image data are shown in Fig. 20 for both etalon systems. The jet exit velocity is indicated for each test case. The actual velocities at the measurement plane, which was approximately 2 jet diameters downstream of the $1 \mathrm{~cm}$ diameter nozzle exit, are expected to be slightly lower than the velocity at the exit. Both etalon velocity profiles exhibit a parabolic flow profile and exhibit reasonable velocity estimates. The error bars in the figure represent the lower bound on the measurement uncertainty based on a Cramer-Rao lower bound uncertainty analysis ${ }^{16}$. The uncertainty in the measurements from the higher order fringes (further away from the center of the fringe pattern) is higher than the lower order fringes since there are less pixels spanning those fringes due to the nonlinear nature of the FP interference pattern in the image space.

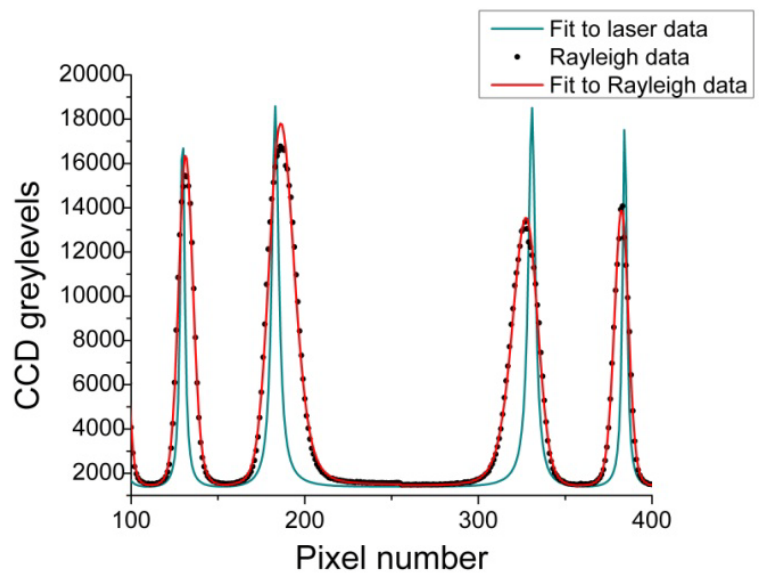

Fig. 18 Linear profile through the center of the Rayleigh and reference fringes for the fused silica etalon Rayleigh experiment showing the data and model function fit to the data.

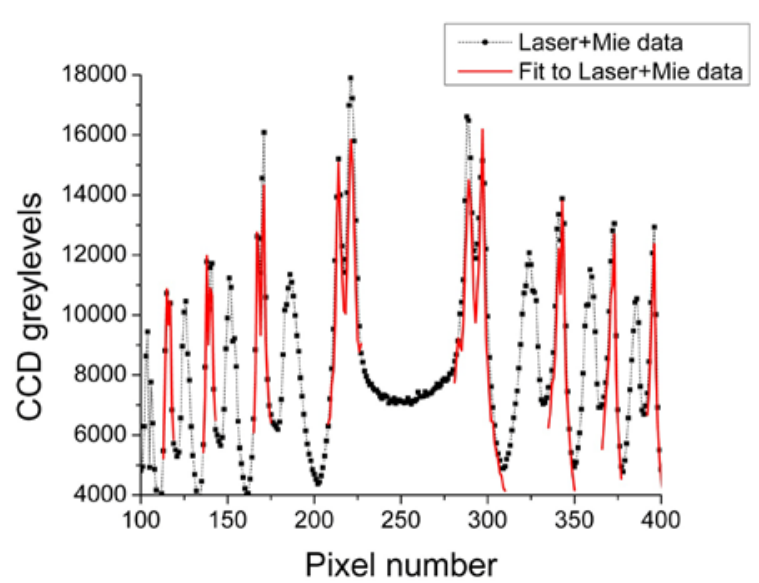

Fig. 19 Linear profile through the center of the reference fringe image for the LCFP etalon Rayleigh experiment showing the data and model function fit to the data.

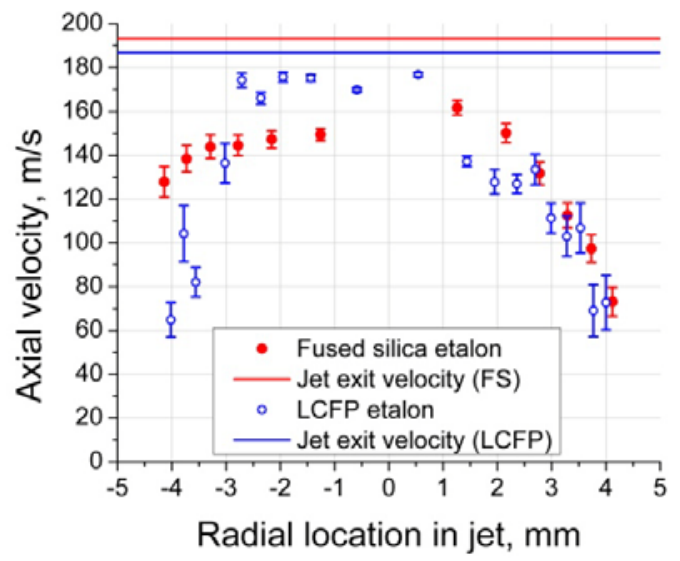

Fig. 20 Radial profile of the axial velocity component in the free jet at an axial plane approximately $2 \mathrm{~cm}$ from the jet exit. The jet exit velocity of the two test cases is indicated in the figure. 


\section{Conclusions and Future Work}

Two etalon systems were characterized to evaluate their usefulness in Rayleigh scattering studies. One etalon was a fused silica solid etalon and the other was a liquid crystal Fabry-Perot (LCFP) etalon, which contained a thin liquid crystal layer in conjunction with glass layers to form a solid etalon cavity. The LCFP etalon demonstrated better short-term apparent frequency stability than the fused silica etalon. The apparent frequency stability of the etalons was of interest since drift in the etalon characteristics can lead to errors in the measured frequency shift, and hence velocity measurements, in Rayleigh scattering experiments. The LCFP exhibited apparent frequency stability of $\pm 2 \mathrm{MHz}$ over a 60 second time period compared to $\pm 4 \mathrm{MHz}$ for the fused silica etalon, corresponding to an error in the calculated velocity of as much as $1.5 \mathrm{~m} / \mathrm{s}$ and $3.0 \mathrm{~m} / \mathrm{s}$, respectively. The temperature sensitivity of the fused silica etalon was measured to be approximately $3600 \mathrm{MHz} /{ }^{\circ} \mathrm{C}$ whereas the temperature sensitivity of the LCFP etalon was measured to be approximately $550 \mathrm{MHz} /{ }^{\circ} \mathrm{C}$. To control the frequency stability of the fused silica etalon in a non-thermally-controlled environment, as is commonly encountered in many test facilities, to $\pm 4 \mathrm{MHz}$ and the LCFP to $\pm 2 \mathrm{MHz}$ would require controlling the etalon temperature to within $0.001{ }^{\circ} \mathrm{C}$ and $0.003{ }^{\circ} \mathrm{C}$ respectively. This proves to be unrealistic since standard temperature controllers are only capable of controlling the temperature within $0.1{ }^{\circ} \mathrm{C}$. It is often useful to control the etalon's physical properties to set the interference fringes to desired locations. For the fused silica etalon this can only be achieved by controlling the etalon temperature, which is cumbersome and has a slow time response. The LCFP can be tuned rapidly by adjusting the voltage applied to the liquid crystal layer, which changes the index of refraction of the medium. This can be done very rapidly (on the order of milliseconds) compared to the slow time response of temperature tuning ( minutes). A plot of fringe location as a function of liquid crystal voltage was presented to show the capability of tuning the LCFP. This capability may be very useful in future work for setting the interference fringes to desired locations or stabilizing the etalon system to reduce velocity measurement error due to changes in the etalon refractive index in environments with significant temperature fluctuations. Finally, both etalon systems were used to acquire Rayleigh scattering data in a free jet with an exit velocity of approximately $190 \mathrm{~m} / \mathrm{s}$. By directly imaging the laser beam in the flow field through each solid etalon system nearly the entire radial profile of the jet flow was acquired in a single image snapshot. The fused silica etalon produced reasonable velocity estimates from the Rayleigh data; however the low free spectral range of the LCFP etalon made analysis of the Rayleigh signal difficult due to overlap between adjacent Rayleigh fringe signals. It was fortunate that some particle (Mie) scattering was recorded in the LCFP experiment that was used to obtain reasonably accurate velocity estimates.

The use of the LCFP in Rayleigh experiments will be investigated further to determine if there are modifications that can be made to the system to make it useable for this purpose. The characteristics of the LCFP will be analyzed further to determine if the fringe quality can be improved by operating the device at different temperature and voltage settings. A LCFP with a larger FSR may be designed for future work.

\section{Acknowledgments}

This work is supported by the Hypersonics discipline under NASA’s Fundamental Aeronautics Program.

\section{References}

\footnotetext{
${ }^{1}$ Seasholtz, R. G., Buggele, A. E., and Reeder, M. F., "Flow Measurements Based on Rayleigh Scattering and Fabry-Perot Interferometer," Optics and Lasers in Engineering, Vol. 27, No. 6, 1997, pp. 543-570.

${ }^{2}$ Seasholtz, R. G., Panda, J., and Elam, K. A., "Rayleigh Scattering Diagnostic for Measurement of Velocity and Density Fluctuation Spectra,” AIAA-2002-0827, 2002.

${ }^{3}$ Mielke, A. F., Seasholtz, R. G., Elam, K.A., and Panda, J., “Time-average Measurement of Velocity, Density, Temperature, and Turbulence Velocity Fluctuations Using Rayleigh and Mie Scattering,” Experiments in Fluids, Vol. 39, No. 2, 2005, 441-

${ }^{4}$ Mielke, A. F., Elam, K. A., and Sung, C. J., "Multiproperty Measurements at High Sampling Rates Using Rayleigh Scattering,” AIAA Journal, Vol. 47, No. 4, 2009, pp. 850-862.

${ }^{5}$ Bivolaru, D., Cutler, A. D., Danehy, P. M., Gaffney, R. L., and Baurle, R. A., "Spatially and Temporally Resolved Measurements of Velocity in a $\mathrm{H}_{2}$-air Combustion-heated Supersonic Jet,” AIAA-2009-27, 2009.

${ }^{6}$ Bivolaru, D., Danehy, P. M., and Cutler, A. D., "Multi-Point Interferometric Rayleigh Scattering Using Dual-pass Light Recirculation,” AIAA-2008-3708, 2008.

${ }^{7}$ Mielke, A. F., Elam, K. A., Clem, M. M., “Multiple-Point Mass Flux Measurement System Using Rayleigh Scattering,” AIAA-2009-528, 2009.

${ }^{8}$ Miles, R. B., Lempert, W. R., and Forkey, J. N., “Laser Rayleigh Scattering,” Meas. Sci. Technol., Vol. 12, No. 5, 2001, pp.
} 454. R33-R51. 
${ }^{9}$ Mielke, A. F., “Development of a Molecular Rayleigh Scattering Diagnostic for Simultaneous Time-Resolved Measurement of Temperature, Velocity, and Density,” Ph.D. Dissertation, Department of Mechanical and Aerospace Engineering, Case Western Reserve Univ., Cleveland, OH, 2008, pp. 170-176.

${ }^{10}$ Vaughan, J. M., The Fabry Perot Interferometer, History, Theory, Practice, and Applications, Adam Hilger, Philadelphia, 1989, pp. 89-134.

${ }^{11}$ Patel, J. S., Saifi, M. A., Berreman, D. W., Lin, C., Andreadakis, N., and Lee, S. D., "Electrically Tunable Optical Filter for Infrared Wavelength Using Liquid Crystals in a Fabry-Perot Etalon,” Applied Physics Letter, Vol. 57, No. 17, 1990, 1718-1720.

${ }^{12}$ Maeda, M. W., Patel, J. S., Lin, C., Horrobin, J., and Spicer, R., "Electrically Tunable Liquid-Crystal-Etalon Filter for High-Density WDM Systems,” IEEE Photonics Technology Letters, Vol. 2, No. 11, 1990, 820-822.

${ }^{13}$ Tenti, G., Boley, C., D., and Desai, R. C., "On the Kinetic Model Description of Rayleigh-Brillouin Scattering from Molecular Gases,” Canadian Journal of Physics, Vol. 52, No. 4, 1974, pp. 285-290.

${ }^{14}$ Boley, C. D., Desai, R. C., and Tenti, G., "Kinetic models and Brillouin scattering in a molecular gas," Canadian Journal of Physics, Vol. 50, No. 18, 1972, pp. 2158-2173.

${ }^{15}$ Edwards, R. V., Processing Random Data: Statistics for Engineers and Scientists, World Scientific, New Jersey, 2006, pp.91-97.

${ }^{16}$ Whalen, A. D., Detection of Signals in Noise, Academic Press, New York, 1971, pp. 327-332. 